\title{
New constraints on axion-mediated P,T-violating interaction from electric dipole moments of diamagnetic atoms
}

\author{
V. A. Dzuba, ${ }^{1}$ V. V. Flambaum, ${ }^{1}$ I. B. Samsonov, ${ }^{1,2}$ and Y. V. Stadnik ${ }^{3}$ \\ ${ }^{1}$ School of Physics, University of New South Wales, Sydney, New South Wales 2052, Australia \\ ${ }^{2}$ Bogoliubov Laboratory of Theoretical Physics, JINR, Dubna, Moscow region 141980, Russia \\ ${ }^{3}$ Helmholtz Institute Mainz, Johannes Gutenberg University, 55099 Mainz, Germany
}

\begin{abstract}
The exchange of an axion-like particle between atomic electrons and the nucleus may induce electric dipole moments (EDMs) of atoms and molecules. This interaction is described by a parityand time-reversal-invariance-violating potential which depends on the product of a scalar $g^{s}$ and a pseudoscalar $g^{p}$ coupling constant. We consider the interaction with the specific combination of these constants, $g_{e}^{s} g_{N}^{p}$, which gives significant contributions to the EDMs of diamagnetic atoms. In this paper, we calculate these contributions to the EDMs of ${ }^{199} \mathrm{Hg},{ }^{129} \mathrm{Xe},{ }^{211} \mathrm{Rn}$ and ${ }^{225} \mathrm{Ra}$ for a wide range of axion masses. Comparing these results with recent experimental EDM measurements, we place new constraints on $g_{e}^{s} g_{N}^{p}$. The most stringent atomic EDM limits come from ${ }^{199} \mathrm{Hg}$ and improve on existing laboratory limits from other experiments for axion masses exceeding $10^{-2} \mathrm{eV}$.
\end{abstract}

\section{INTRODUCTION}

In field theory, the interaction of the axion field $a$ with fermions $\psi$ may be described by the Lagrangian density

$$
\mathcal{L}_{\mathrm{int}}=a \sum_{\psi} \bar{\psi}\left(g_{\psi}^{s}+i g_{\psi}^{p} \gamma_{5}\right) \psi
$$

where $g_{\psi}^{s}$ and $g_{\psi}^{p}$ are model-dependent coupling constants and $\gamma_{5}=-i \gamma_{0} \gamma_{1} \gamma_{2} \gamma_{3}$ in the notation of [1] for Dirac matrices. This Lagrangian appears naturally in the case of the canonical axion, which solves the strong $\mathrm{CP}$ problem of quantum chromodynamics [2 8]. In Eq. (1), we assume, however, a generic axion-like particle, which couples to different fermions with independent constants $g_{\psi}^{s}$ and $g_{\psi}^{p}$. Consistency with various experimental data imposes very severe constraints on different combinations of such couplings, see, e.g., Ref. 9] for a review. Since these interactions are extremely weak, the axion can naturally be considered a candidate for dark matter [10 12 .

In atomic phenomena, the interaction (1) implies the exchange of an axion between the atomic electrons and the nucleus described by the P,T-violating potential

$$
V(r)=i \frac{g^{p} g^{s}}{4 \pi} \frac{e^{-m_{a} r}}{r} \gamma^{0} \gamma_{5}
$$

where $m_{a}$ is the axion mass. In Ref. 13, it was shown that this potential induces anomalous contributions to EDMs in atoms and molecules due to mixing of atomic states of opposite parity. The comparison of these EDMs with the corresponding experimentally observed values imposes strong constraints on the coupling constants $g^{s}$ and $g^{p}$ of the interaction (1).

Ref. 13] considered the case when the pseudoscalar interaction constant $g^{p} \equiv g_{e}^{p}$ is attributed to the electron, while the scalar interaction constant $g^{s} \equiv g_{N}^{s}$ corresponds to either another electron or a nucleon. In the latter case, the potential (2) reduces (in the non-relativistic limit) to

$$
V(r)=-\frac{g_{e}^{p} g_{N}^{s}}{8 \pi m_{e}} \boldsymbol{\Sigma} \cdot \boldsymbol{\nabla}\left(\frac{e^{-m_{a} r}}{r}\right),
$$

where $m_{e}$ is the electron mass and $\boldsymbol{\Sigma}=\left(\begin{array}{cc}\boldsymbol{\sigma} & 0 \\ 0 & \boldsymbol{\sigma}\end{array}\right)$ is the Dirac spin matrix vector acting on the electron wavefunctions. Analysis of contributions to atomic EDMs due to the potential (3) allowed the authors to place constraints on the product of coupling constants $g_{e}^{p} g_{N}^{s}$. The constraints derived in Ref. [13] gave a significant improvement over previous laboratory limits on these interaction constants for certain axion masses [14 20.

In this paper, we consider the opposite case, namely when the constant $g^{s} \equiv g_{e}^{s}$ corresponds to the interaction of the axion with an electron, while $g^{p} \equiv g_{N}^{p}$ corresponds to the interaction with a nucleon. In this case, the potential (2) reduces to the following form

$$
V(r)=-\frac{g_{e}^{s} g_{N}^{p}}{8 \pi m_{N}} \boldsymbol{\sigma}_{N} \cdot \nabla\left(\frac{e^{-m_{a} r}}{r}\right) \gamma^{0},
$$

where $m_{N}$ and $\boldsymbol{\sigma}_{N}$ are the nucleon mass and its spin unit vector, respectively. The Dirac matrix $\gamma^{0}$ corresponds to the atomic electrons. The potential (4) will allow us to place new constraints on the combination of coupling constants $g_{e}^{s} g_{N}^{p}$, which is independent from the case considered in 13 .

Although the potentials (3) and (4) look similar, they manifest themselves differently in atomic phenomena. The potential (3) describes the interaction of electron's spin with the nuclear density. Thus, this interaction may give significant contributions to the atomic EDMs of paramagnetic atoms with open electron shells. On the other hand, the potential (4) is responsible for the interaction of the nuclear spin with the electron density, which may contribute significantly to the atomic EDMs of diamagnetic atoms with closed electron shells, but with non-zero nuclear spins. Therefore, we perform numerical calculations of the corresponding EDMs for atomic ${ }^{129} \mathrm{Xe},{ }^{199} \mathrm{Hg},{ }^{211} \mathrm{Rn}$ and ${ }^{225} \mathrm{Ra}$ to interpret existing experimental data. We find that the most stringent constraint arises from the recent EDM measurement in ${ }^{199} \mathrm{Hg}$ 21]:

$$
d\left({ }^{199} \mathrm{Hg}\right)=\left(2.20 \pm 2.75_{\text {stat }} \pm 1.48_{\text {syst }}\right) \times 10^{-30} e \mathrm{~cm} .
$$


This allows us to place new bounds on the combination of coupling constants $g_{e}^{s} g_{N}^{p}$ for a wide range of axion masses. Measurements of EDMs in the other diamagnetic atoms 22 24] give less stringent constraints.

We note that in the limit of a large axion mass, the potential (4) reduces to the following contact interaction

$$
\lim _{m_{a} \rightarrow \infty} m_{a}^{2} V(r)=-\frac{g_{e}^{s} g_{N}^{p}}{2 m_{N}} \boldsymbol{\sigma}_{N} \cdot \nabla\left[\delta^{3}(\mathbf{r})\right] \gamma^{0} .
$$

In field theory, this potential corresponds to the parityand time-reversal-invariance-violating four-fermion interaction Lagrangian density

$$
\mathcal{L}=-\frac{G_{F}}{\sqrt{2}} C_{P S} \bar{N} i \gamma_{5} N \bar{e} e
$$

where $N$ and $e$ denote the nucleon and electron fields, respectively; $G_{F}$ is the Fermi constant and $C_{P S}=$ $-\sqrt{2} g_{e}^{s} g_{N}^{p} /\left(G_{F} m_{a}^{2}\right)$. The contributions to the EDMs of diamagnetic atoms due to this operator were studied in previous works 25,26 . In the next section, we extend these earlier calculations to the potential (4), which is defined for an arbitrary axion mass.

\section{CALCULATIONS AND RESULTS}

The potential (4) describes the interaction of a nonpolarized electron with a polarized nucleon with spin $\boldsymbol{\sigma}_{N}$. To apply this potential to the electron-nucleus interaction in an atom, we have to average it over the atomic nucleus,

$$
\bar{V}=-\frac{g_{e}^{s} g_{N}^{p}}{8 \pi m_{N}}\left\langle\boldsymbol{\sigma}_{N}\right\rangle \cdot\left\langle\boldsymbol{\nabla}\left(\frac{e^{-m_{a} r}}{r}\right)\right\rangle \gamma^{0} .
$$

Here, the quantity $\left\langle\boldsymbol{\sigma}_{N}\right\rangle$ is proportional to the total angular momentum of the nucleus $\mathbf{I}$

$$
\left\langle\boldsymbol{\sigma}_{N}\right\rangle=\kappa \mathbf{I} / I
$$

For spherically symmetric non-excited nuclei ${ }^{129} \mathrm{Xe}$ and ${ }^{199} \mathrm{Hg}$ the coefficient $\kappa$ can be computed within the Schmidt (single-particle approximation) model [26, 27] (see also Refs. 28 30, which employ more sophisticated nuclear models). For the deformed nuclei ${ }^{211} \mathrm{Rn}$ and ${ }^{225} \mathrm{Ra}$ it is appropriate to use the Nilsson nuclear model (see, e.g., 31]). We collect the resulting values of the coefficient $\kappa$ in Table I. We point out that the spins of these nuclei are predominantly due to the spin of the unpaired valence neutron, irrespective of the nuclear model used. Thus, experimental measurements of EDMs of these atoms are mainly sensitive to the parameter $g_{n}^{p}$, which corresponds to the interaction of an axion with a neutron.

The potential (8) involves the Yukawa-type interaction which should be averaged over the nuclear density $\rho(\mathbf{R})$,

$$
\left\langle\frac{e^{-m_{a} r}}{r}\right\rangle=\int d^{3} \mathbf{R} \frac{e^{-m_{a}|\mathbf{r}-\mathbf{R}|}}{|\mathbf{r}-\mathbf{R}|} \rho(\mathbf{R}) .
$$

TABLE I. Values of the coefficient $\kappa$ in Eq. (9) for different nuclei. For spherically symmetric nuclei ${ }^{129} \mathrm{Xe}$ and ${ }^{199} \mathrm{Hg}$ this coefficient is found using the Schmidt nuclear model while for the deformed nuclei ${ }^{211} \mathrm{Rn}$ and ${ }^{225} \mathrm{Ra}$ the Nilsson deformed oscillator model is applied.

\begin{tabular}{c|c|c|c|c}
\hline \hline & ${ }^{129} \mathrm{Xe}$ & ${ }^{199} \mathrm{Hg}$ & ${ }^{211} \mathrm{Rn}$ & ${ }^{225} \mathrm{Ra}$ \\
\hline$\kappa$ & +1 & $-\frac{1}{3}$ & $-\frac{1}{3}$ & $\frac{1}{3}$ \\
\hline \hline
\end{tabular}

Note that, according to the experimental data discussed in Ref. 1], the shape of the nuclear spin density is very close to the shape of the nuclear charge density. The nuclear density is well described by the Fermi function

$$
\rho(\mathbf{R})=\frac{\rho_{0}}{1+\exp \left(\frac{R-R_{0}}{a}\right)},
$$

where $R_{0}$ and $a$ are nucleus-dependent parameters and $\rho_{0}$ is normalized according to $\int \rho(\mathbf{R}) d^{3} \mathbf{R}=1$. The values of these parameters for various isotopes are tabulated, e.g., in 32. The potential (8), averaged over the nuclear density (11), takes the following radial form

$$
\begin{aligned}
& V_{r}(r)=-\frac{\kappa G_{F} C_{P S} m_{a}^{3}}{2 \sqrt{2} m_{N}}\left[-\frac{e^{-m_{a} r}}{m_{a}^{2} r^{2}}\left(1+m_{a} r\right)\right. \\
& \times \int_{0}^{r} r^{\prime} \rho\left(r^{\prime}\right) \sinh \left(m_{a} r^{\prime}\right) d r^{\prime}+\frac{1}{m_{a}^{2} r^{2}}\left[m_{a} r \cosh \left(m_{a} r\right)\right. \\
& \left.\left.-\sinh \left(m_{a} r\right)\right] \int_{r}^{\infty} r^{\prime} \rho\left(r^{\prime}\right) e^{-m_{a} r^{\prime}} d r^{\prime}\right]
\end{aligned}
$$

With the single-electron wave function of the form

$$
\psi(\mathbf{r})=\frac{1}{r}\left(\begin{array}{c}
f(r) \Omega_{j l m} \\
i g(r) \Omega_{j \tilde{l m}}
\end{array}\right),
$$

where $\Omega_{j l m}$ is the spherical spinor, the single-electron matrix element of the operator $(8)$ is given by

$$
\begin{aligned}
& \langle i\|\bar{V}\| j\rangle=\left\langle\kappa_{i}\left\|C^{(1)}\right\| \kappa_{j}\right\rangle \times \\
& \int V_{r}(r)\left[f_{i}(r) f_{j}(r)-g_{i}(r) g_{j}(r)\right] d r
\end{aligned}
$$

where $\kappa=(-1)^{j+1 / 2-l}(j+1 / 2)$ is the angular quantum number which determines the angular momentum $l$ and total angular momentum $j$; the potential $V(r)$ is given by 12 , and $C^{(1)}$ is the normalised spherical function of first rank.

The interaction potential (8) induces an atomic EDM which in the leading order in perturbation theory reads

$$
\mathbf{d}=2 \sum_{M} \frac{\langle 0|\bar{V}| M\rangle\langle M|-e \mathbf{r}| 0\rangle}{E_{0}-E_{M}}
$$

where $e$ is the electron charge and the summation is over the complete set of excited states $|M\rangle$ with energies $E_{M}$. Note that if the operator $\bar{V}$ is replaced in 15 by the electric dipole operator $-e \mathbf{r}$, then this expression gives 
the scalar static polarizability of the atom. This can be used to check the accuracy of the calculations.

Eq. (15) is exact if $|0\rangle$ and $|M\rangle$ are exact manyelectron wave functions of the whole atom. In practice, we need to reduce the calculations to single-electron matrix elements while including many-body effects as corrections to the wave function or to the operator. In the present work, we use the self-consistent relativistic DiracHartree-Fock method in the external field, also known as the random-phase approximation (RPA). This way we include the electron core polarization corrections to the operator of the external field. All electron states from the closed shells are treated as the core states producing the Hartree-Fock potential. In the closed-shell atoms which we consider in this paper, all electrons are included in the electron core.

The RPA equations can be written in terms of the corrections to all electron wave functions induced by the external field

$$
\left(\hat{H}^{\mathrm{HF}}-\epsilon_{c}\right) \delta \psi_{c}=-\left(\hat{F}+\delta V^{F}\right) \psi_{c} .
$$

Here $\hat{H}^{\mathrm{HF}}$ is the relativistic Hartree-Fock operator, $\psi_{c}$ is the single-electron wave function for a state $c, \hat{F}$ is the operator of the external field (either electric dipole $\mathbf{D}=-e \mathbf{r}$ or parity-violating operator (8p), $\delta V^{F}$ is the correction to the self-consistent Hartree-Fock potential (including the exchange interaction) due to the change of all electron states imposed by the external field. Equations (16) are solved self-consistently for all states in the core. After that, the EDM of a closed-shell atom in the RPA approximation is given by

$$
\mathbf{d}=\frac{2}{3} \sum_{c}\left\langle\psi_{c}\|\bar{V}\| \delta \psi_{c}^{d}\right\rangle \equiv \frac{2}{3} \sum_{c}\left\langle\psi_{c}\|\mathbf{D}\| \delta \psi_{c}^{\bar{V}}\right\rangle .
$$

Here $\delta \psi_{c}^{d}$ comes from solving the RPA equations 16 with the electric dipole operator $(\hat{F}=\mathbf{D})$, while $\delta \psi_{c}^{V}$ comes from solving the RPA equations with the operator $\bar{V}$. The two equations in (17) are equivalent and comparing the results can be used to check computer codes. Note that it is sufficient to solve the RPA equations only once with either of the two operators. As in Eq. (15), replacing the $\bar{V}$ operator in 177 by the electric dipole operator gives the scalar dipole polarizability of the atom in the RPA approximation. It is known that the RPA approximation give very good accuracy for the polarizabilities of noble-gas atoms (see, e.g. 33). The difference with experiment is at the level of $1-4 \%$ for $\mathrm{Kr}$ and Xe. On the other hand, the difference is larger for atoms like $\mathrm{Hg}$ and Ra. These atoms have $6 s^{2}$ and $7 s^{2}$ outermost subshells. Therefore, we can still treat them as closed-shell systems and use (16) and (17) for the calculations. The difference with experiment is $7 \%$ for the polarizability of $\mathrm{Ra}$ and $25 \%$ for the polarizability of $\mathrm{Hg}$ [33. This difference between calculated and measured polarizabilities is mostly due to many-body effects beyond the core polarization. This is the dominating source of uncertainty in the present atomic calculations. The contribution of these correlations to the EDM of $\mathrm{Hg}, \mathrm{Yb}, \mathrm{Ra}$ and other atoms was studied in detail for a range of singular operators in our earlier works 26, 34. It was found that this contribution does not exceed $15 \%$. Other factors, like numerical accuracy in calculating the potential $(12)$ or solving the RPA equations (16), or uncertainty in the nuclear parameters in (11), among others, give much smaller contributions to the uncertainties. Thus we conclude that our accuracy for the atomic EDM calculations is within $10 \%$ for $\mathrm{Xe}$ and $\mathrm{Rn}$ and $30 \%$ for $\mathrm{Hg}$ and $\mathrm{Ra}$.

The results of calculations are summarized in Table I. In this table, the results for an infinite axion mass are taken from [26, where the EDMs induced by the operator (6) were calculated.

TABLE II. Summary of relativistic Hartree-Fock-Dirac calculations of atomic EDMs induced by the interaction (8) for various axion masses. These values are given in the units $C_{P S} \cdot \kappa \cdot e \cdot \mathrm{cm}$, where $C_{P S}=-\sqrt{2} g_{e}^{s} g_{N}^{p} /\left(G_{F} m_{a}^{2}\right)$. These calculations take into account the effects of all atomic electrons. For axion masses $m_{a} \ll 1 \mathrm{keV}$, the interaction (8) becomes long-range and the induced atomic EDMs become independent of $m_{a}$. The estimated accuracy is within $10 \%$ for Xe and $\mathrm{Rn}$ and $30 \%$ for $\mathrm{Hg}$ and $\mathrm{Ra}$.

\begin{tabular}{c|c|c|c|c}
\hline \hline $\begin{array}{c}m_{a} \\
(\mathrm{eV})\end{array}$ & ${ }^{129} \mathrm{Xe}$ & ${ }^{199} \mathrm{Hg}$ & ${ }^{211} \mathrm{Rn}$ & ${ }^{225} \mathrm{Ra}$ \\
\hline$\infty$ & $1.6 \times 10^{-23}$ & $-1.8 \times 10^{-22}$ & $2.1 \times 10^{-22}$ & $-6.4 \times 10^{-22}$ \\
$10^{8}$ & $1.4 \times 10^{-23}$ & $-1.8 \times 10^{-22}$ & $1.7 \times 10^{-22}$ & $-5.2 \times 10^{-22}$ \\
$10^{7}$ & $3.6 \times 10^{-24}$ & $-3.7 \times 10^{-23}$ & $3.5 \times 10^{-23}$ & $-1.0 \times 10^{-22}$ \\
$10^{6}$ & $5.4 \times 10^{-25}$ & $-2.4 \times 10^{-24}$ & $2.1 \times 10^{-24}$ & $-5.4 \times 10^{-24}$ \\
$10^{5}$ & $8.9 \times 10^{-27}$ & $-2.7 \times 10^{-26}$ & $1.7 \times 10^{-26}$ & $-5.5 \times 10^{-26}$ \\
$10^{4}$ & $4.2 \times 10^{-29}$ & $-2.0 \times 10^{-28}$ & $1.5 \times 10^{-28}$ & $-4.5 \times 10^{-28}$ \\
$10^{3}$ & $1.1 \times 10^{-30}$ & $-1.0 \times 10^{-30}$ & $2.1 \times 10^{-30}$ & $-3.7 \times 10^{-30}$ \\
$10^{2}$ & $1.2 \times 10^{-32}$ & $-7.8 \times 10^{-33}$ & $2.3 \times 10^{-32}$ & $-3.1 \times 10^{-32}$ \\
10 & $1.2 \times 10^{-34}$ & $-7.8 \times 10^{-35}$ & $2.3 \times 10^{-34}$ & $-3.1 \times 10^{-34}$ \\
\hline \hline
\end{tabular}

TABLE III. Asymptotic values of EDMs of atoms for low and high axion mass. The values in this table originate from the corresponding values in Table II after substitution of the value of the Fermi coupling constant $G_{F} \approx 1.167 \times 10^{-5} \mathrm{GeV}^{-2}$ and the coefficient $\kappa$ from Table 1

\begin{tabular}{c|c|c}
\hline \hline$|d|, e \cdot \mathrm{cm}$ & $m_{a} \lesssim 10^{3} \mathrm{eV}$ & $m_{a} \gtrsim 10^{8} \mathrm{eV}$ \\
\hline${ }^{129} \mathrm{Xe}$ & $1.5 \times 10^{-13} g_{e}^{s} g_{N}^{p}$ & $1.7 g_{e}^{s} g_{N}^{p}\left(\frac{\mathrm{eV}}{m_{a}}\right)^{2}$ \\
\hline${ }^{199} \mathrm{Hg}$ & $3.2 \times 10^{-14} g_{e}^{s} g_{N}^{p}$ & $7.3 g_{e}^{s} g_{N}^{p}\left(\frac{\mathrm{eV}}{m_{a}}\right)^{2}$ \\
\hline${ }^{211} \mathrm{Rn}$ & $9.3 \times 10^{-14} g_{e}^{s} g_{N}^{p}$ & $8.5 g_{e}^{s} g_{N}^{p}\left(\frac{\mathrm{eV}}{m_{a}}\right)^{2}$ \\
\hline${ }^{225} \mathrm{Ra}$ & $1.3 \times 10^{-13} g_{e}^{s} g_{N}^{p}$ & $25 g_{e}^{s} g_{N}^{p}\left(\frac{\mathrm{eV}}{m_{a}}\right)^{2}$ \\
\hline \hline
\end{tabular}

\section{DISCUSSION}

In Table II w we present the results of our computations of the EDMs in four diamagnetic atoms $\left({ }^{129} \mathrm{Xe},{ }^{199} \mathrm{Hg}\right.$, 


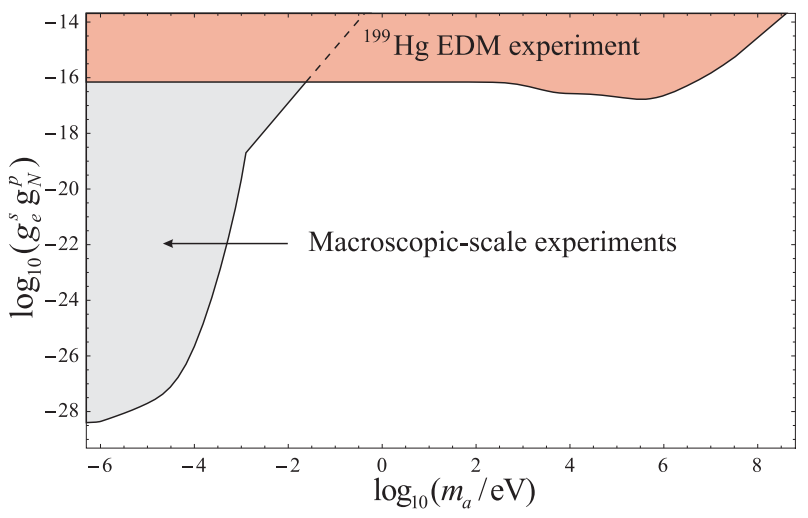

FIG. 1. Laboratory constraints on the parity- and timereversal-invariance-violating scalar-pseudoscalar electronnucleon interaction mediated by an axion of mass $m_{a}$. The pink exclusion region is the result of this work. The gray exclusion region summarizes the combined results which were derived from the earlier macroscopic-scale experiments [35 39] with graphical accuracy.

${ }^{211} \mathrm{Rn}$ and ${ }^{225} \mathrm{Ra}$ ) induced by the P,T-odd potential (4). In Table III. we also collect the asymptotical values of EDMs for these atoms at low $\left(m_{a} \lesssim 10^{3} \mathrm{eV}\right)$ and high $\left(m_{a} \gtrsim 10^{8} \mathrm{eV}\right)$ axion masses. Combining these results with experimental measurements of EDMs in these atoms imposes constraints on the product of coupling constants $g_{e}^{s} g_{N}^{p}$. The most stringent constraint comes from the ${ }^{199} \mathrm{Hg}$ EDM experiment [21] given in Eq. (5) and is shown in Fig. 1 by the pink exclusion region. The pink exclusion region in Fig. 1 possesses the following asymptotics:

$$
\begin{array}{ll}
m_{a} \lesssim 10^{3} \mathrm{eV} & \left|g_{e}^{s} g_{N}^{p}\right|<7 \times 10^{-17} \\
m_{a} \gtrsim 10^{8} \mathrm{eV} & \left|g_{e}^{s} g_{N}^{p}\right|<3 \times 10^{-31}\left(\frac{m_{a}}{\mathrm{eV}}\right)^{2}
\end{array}
$$

The latter constraint originates from the results of the paper [26], where the atomic EDMs due to the operator (6) were studied.

It is interesting to compare our results with earlier constraints from macroscopic-scale experiments 35 39, which reported constraints on the coupling parameters $g_{N}^{s} g_{n}^{p}$, where $g_{n}^{p}$ denotes the axion coupling to a polarized neutron, while $g_{N}^{s}$ denotes the coupling to the nucleons in a non-polarized massive body. Let $\langle A\rangle$ and $\langle Z\rangle$ be the average atomic mass and proton numbers in the non-polarized massive body, respectively. Then, in general, the polarized neutron interacts with a nonpolarized atom through the combination of constants $\left(g_{e}^{s}\langle Z\rangle+g_{N}^{s}\langle A\rangle\right) g_{n}^{p}$. The constraints on $g_{N}^{s} g_{n}^{p}$ were obtained in [35]39] with the assumption $\langle A\rangle\left|g_{N}^{s}\right| \gg\langle Z\rangle\left|g_{e}^{s}\right|$, but we can assume the opposite case, $\langle A\rangle\left|g_{N}^{s}\right| \ll\langle Z\rangle\left|g_{e}^{s}\right|$, to find the constraints on $g_{e}^{s} g_{n}^{p}$. Since different experiments deal with different materials, we make the simple approximation $\langle A\rangle /\langle Z\rangle \approx 2.2$. This allows us to represent the results of the earlier works 35 39 in the form of the gray exclusion region in Fig. 1. We conclude that our results give significantly improved laboratory limits on $g_{e}^{s} g_{N}^{p}$ for $m_{a} \gtrsim 10^{-2} \mathrm{eV}$.

We note that there are more stringent indirect bounds from the combination of stellar energy-loss arguments and laboratory searches for spin-independent fifth forces [40] or from the combination of stellar energy-loss arguments in several different astrophysical systems [41-44] for certain axion masses, though astrophysical bounds may be evaded by mechanisms that inhibit the processes of stellar "cooling" via axion emission 45 47. Finally, we mention that limits on the nucleon-nucleon interaction constants $g_{N}^{s} g_{N^{\prime}}^{p}$ have been derived from the consideration of the nuclear Schiff moments induced by the exchange of a low-mass axion-like particle between nucleons within a nucleus 48].

Acknowledgments. The authors are grateful to Bryce Lackenby for useful comments. This work is supported by the Australian Research Council Grant No. PD150101405. V.V.F. acknowledges support from the Gutenberg Fellowship and New Zealand Institute for Advanced Studies. Y.V.S. was supported by the Humboldt Research Fellowship.
[1] I. B. Khriplovich, Parity Nonconservation in Atomic Phenomena, Gordon and Breach Science Publishers, Philadelphia, 1991.

[2] R. D. Peccei and H. R. Quinn, Phys. Rev. Lett. 381440 (1977).

[3] S. Weinberg, Phys. Rev. Lett. 40, 223 (1978).

[4] F. Wilczek, Phys. Rev. Lett. 40, 279 (1978).

[5] J. E. Kim, Phys. Rev. Lett. 43, 103 (1979).

[6] M. A. Shifman, A. I. Vainshtein and V. I. Zakharov, Nucl. Phys. B 166, 493 (1980).

[7] A. R. Zhitnitsky, Sov. J. Nucl. Phys. 31, 529 (1980), [Yad. Fiz. 31, 1024 (1980)].

[8] M. Dine, W. Fischler and M. Srednicki, Phys. Lett. 104B 199 (1981).

[9] M. S. Safronova, D. Budker, D. DeMille, D. F. J. Kimball, A. Derevianko and C. W. Clark, (2017),
arXiv: 1710.01833

[10] J. Preskill, M. B. Wise and F. Wilczek, Phys. Lett. 120B, 127 (1983).

[11] L. F. Abbott and P. Sikivie, Phys. Lett. 120B, 133 (1983).

[12] M. Dine and W. Fischler, Phys. Lett. 120B, 137 (1983).

[13] Y. V. Stadnik, V. A. Dzuba and V. V. Flambaum, Phys. Rev. Lett. 120, 013202 (2018).

[14] A. N. Youdin, D. Krause, Jr., K. Jagannathan, L. R. Hunter, and S. K. Lamoreaux, Phys. Rev. Lett. 77, 2170 (1996).

[15] W.-T. Ni, S.-S. Pan, H.-C. Yeh, L.-S. Hou, and J. Wan, Phys. Rev. Lett. 82, 2439 (1999).

[16] G. D. Hammond, C. C. Speake, C. Trenkel, and A. P. Paton, Phys. Rev. Lett. 98, 081101 (2007).

[17] S. A. Hoedl, F. Fleischer, E. G. Adelberger, and 
B. R. Heckel, Phys. Rev. Lett. 106, 041801 (2011).

[18] W. A. Terrano, E. G. Adelberger, J. G. Lee, and B. R. Heckel, Phys. Rev. Lett. 115, 201801 (2015).

[19] N. Crescini, C. Braggio, G. Carugno, P. Falferi, A. Ortolan, and G. Ruoso, Phys. Lett. B 773, 677 (2017).

[20] X. Rong et al., Nature Commun. 9, 739 (2018).

[21] B. Graner, Y. Chen, E. G. Lindahl and B. R. Heckel, Phys. Rev. Lett. 116, 161601 (2016), [Erratum: Phys. Rev. Lett. 119, 119901 (2017)].

[22] W. Heil, talk at SSP2018, 7th International Symposium on Symmetries in Subatomic Physics (SSP2018), Aachen, 10-15 June 2018.

[23] M. A. Rosenberry and T. E. Chupp, Phys. Rev. Lett. 86, 22 (2001).

[24] M. Bishof et al., Phys. Rev. C 94, 025501 (2016).

[25] V. V. Flambaum and I. B. Khriplovich, Sov. Phys. JETP 62, 872 (1985), [Zh. Eksp. Teor. Fiz. 89, 1505 (1985)]

[26] V. A. Dzuba, V. V. Flambaum and S. G. Porsev, Phys. Rev. A 80, 032120 (2009).

[27] Y. V. Stadnik and V. V. Flambaum, Eur. Phys. J. C 75, 110 (2015).

[28] N. Yoshinaga, K. Higashiyama, R. Arai and E. Teruya, Phys. Rev. C 89, 045501 (2014).

[29] N. Yamanaka, B. K. Sahoo, N. Yoshinaga, T. Sato, K. Asahi and B. P. Das, Eur. Phys. J. A 53, 54 (2017).

[30] K. Yanase, N. Yoshinaga, K. Higashiyama and N. Yamanaka, arXiv:1805.00419.

[31] R. F. Casten, Nuclear Structure From A Simple Perspective, Oxford Univ. Press, New York \& Oxford, 1990.

[32] G. Fricke and K. Heilig, Nuclear Charge Radii, Springer, Berlin and Heidelberg, 2004.
[33] V. A. Dzuba, Phys. Rev. A 93, 032519 (2016).

[34] V. A. Dzuba, V. V. Flambaum, J. S. M. Ginges, and M. G. Kozlov, Phys. Rev. A 66, 012111 (2002).

[35] A. Serebrov, Phys. Lett. B 680, 423 (2009).

[36] A. K. Petukhov, G. Pignol, D. Jullien and K. H. Andersen, Phys. Rev. Lett. 105, 170401 (2010).

[37] K. Tullney, F. Allmendinger, M. Burghoff, W. Heil, S. Karpuk, W. Kilian, S. Knappe-Grüneberg, W. Müller, U. Schmidt, A. Schnabel, F. Seifert, Y. Sobolev and L. Trahms, Phys. Rev. Lett. 111, 100801 (2013).

[38] M. Bulatowicz, R. Griffith, M. Larsen, J. Mirijanian, C. B. Fu, E. Smith, W. M. Snow, H. Yan and T. G. Walker, Phys. Rev. Lett. 111, 102001 (2013).

[39] S. Afach et al., Phys. Lett. B745, 58 (2015).

[40] G. Raffelt, Phys. Rev. D 86, 015001 (2012).

[41] G. G. Raffelt, Phys. Rep. 198, 1 (1990).

[42] G. G. Raffelt, in Axions: Theory, Cosmology, and Experimental Searches, edited by M. Kuster, G. Raffelt, and B. Beltran (Springer, Berlin, 2008), pp. 5171.

[43] E. Hardy and R. Lasenby, JHEP 2, 33 (2017).

[44] J. H. Chang, R. Essig, and S. D. McDermott, arXiv:1803.00993

[45] E. Masso and J. Redondo, JCAP 0509, 015 (2005).

[46] P. Jain and S. Mandal, Int. J. Mod. Phys. D 15, 2095 (2006).

[47] J. Jaeckel, E. Masso, J. Redondo, A. Ringwald, and F. Takahashi, arXiv:hep-ph/0605313.

[48] S. Mantry, M. Pitschmann and M. J. Ramsey-Musolf, Phys. Rev. D 90, 054016 (2014). 